\title{
Strawman redux: management of end-tidal gases in patients at risk of perioperative neurocognitive disorder
}

\author{
W. Alan C. Mutch, MD (1) · Renée M. El-Gabalawy, PhD • M. Ruth Graham, MD
}

Received: 6 May 2020/Revised: 7 July 2020/ Accepted: 9 July 2020/Published online: 22 July 2020

(C) Canadian Anesthesiologists' Society 2020

\section{To the Editor,}

In a previous published commentary in the Journal ${ }^{1}$ entitled "Anesthesia and postoperative delirium: the agent is a strawman-the problem is $\mathrm{CO}_{2}$ ", we proposed that the conduct of anesthesia and, in particular, end-tidal (ET) carbon dioxide $\left(\mathrm{CO}_{2}\right)$ management may be an important contributor to cognitive dysfunction after surgery, now termed perioperative neurocognitive disorder (POND). ${ }^{2}$ Subsequent work by our group and others has confirmed an association between intraoperative hypocapnia and one of the sub-categories of POND-postoperative delirium (POD). ${ }^{3,4}$ Not considered in these publications was the potential influence of the other ET respiratory gases, specifically $\mathrm{O}_{2}$ or potential synergistic interactions between $\mathrm{ETCO}_{2}$ and $\mathrm{ETO}_{2}$ as additional contributing factors to the development of POD, although periods of both hypocapnia and hyperoxia are commonly observed during mechanical ventilation in the course of most routine anesthetics.

We have recently examined the combined effects of hyperoxia and hypocapnia on cerebral blood flow (CBF). ${ }^{5}$ Using pseudo-continuous arterial spin labelling, we have identified marked, dynamic changes in CBF during tightly

W. A. C. Mutch, MD $(\bowtie) \cdot$ M. Ruth Graham, MD Department of Anesthesiology, Perioperative and Pain Medicine, Max Rady College of Medicine, Rady Faculty of Health Sciences, University of Manitoba, Winnipeg, MB, Canada e-mail: wacmutch@shaw.ca

R. M. El-Gabalawy, PhD

Department of Anesthesiology, Perioperative and Pain Medicine, Max Rady College of Medicine, Rady Faculty of Health

Sciences, University of Manitoba, Winnipeg, MB, Canada

Department of Clinical Health Psychology, Max Rady College of Medicine, Rady Faculty of Health Sciences, University of Manitoba, Winnipeg, MB, Canada controlled changes in $\mathrm{ETCO}_{2}$ and $\mathrm{ETO}_{2}$ that are observed commonly during anesthesia. That study also showed the independent effect of hyperoxia at normocapnic values on regional decreases in CBF. Additionally, a synergistic decrement in CBF is described with combined hyperoxia and hypocapnia. Further analysis of these data in Figure (A-C) shows the change in $\mathrm{CBF}$ in a single subject under different test conditions. These conditions were (A) baseline (normoxia/normocapnia) and (B) spontaneous hyperventilation to lower the $\mathrm{ETCO}_{2}$ to 5 $\mathrm{mmHg}$ below baseline in the presence of hyperoxia $\left(\mathrm{ETO}_{2}\right.$ $=400 \mathrm{mmHg}$ ). The difference map (image $2-$ image 1 ) is presented in (C) and decreasing CBF is represented as a colour change from blue to purple. A marked global decrement with combined hyperoxia and hypocapnia is evident with regional grey matter flow frequently decreasing by approximately $50 \%$. As such changes in ET respiratory gases are routinely observed during the course of standard anesthetic care, the magnitude of the resultant changes in CBF may be considered a potential contributory factor to POD. Control of end-tidal gases may be important, but a multicentre retrospective analysis of over 300,000 non-cardiac surgical patients suggests clinicians have not reached consensus on appropriate goals. ${ }^{6}$ After controlling for patient and procedural factors, the median $\mathrm{ETCO}_{2}$ was consistently less than 40 $\mathrm{mmHg}$ with large inter-hospital and inter-provider variability.

We believe that these preliminary findings raise an important issue regarding research on delineating the mechanisms of POD. With access to breath-to-breath monitoring of ET respiratory gases, anesthesiologists are uniquely positioned to comprehensively examine these potential intraoperative drivers of POD. Such high-fidelity data should be incorporated and controlled for in studies 


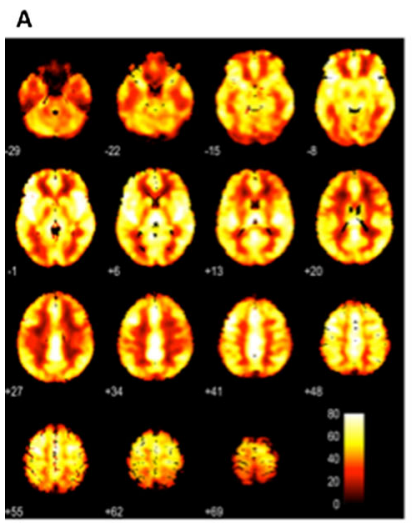

Baseline - Normoxia/Normocapnia (NN)

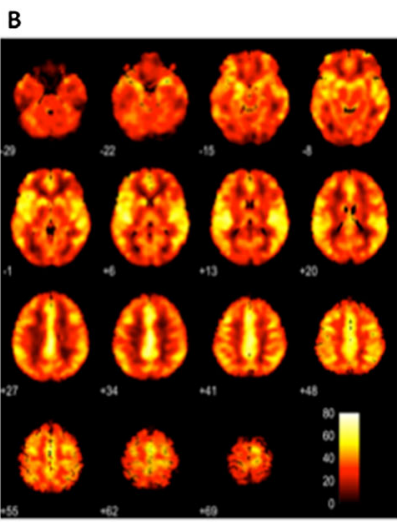

Hyperoxia/Hypocapnia (HH)

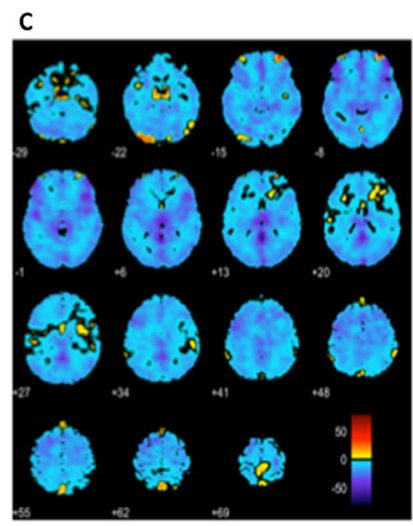

Difference Map: $\mathrm{HH}-\mathrm{NN}$
Figure (A-C) Images from a healthy, awake subject comparing the $\mathrm{CBF}$ at baseline, i.e., normocapnia/normoxia (A). (B) The same patient after being instructed to spontaneously hyperventilate, decreasing the $\mathrm{ETCO}_{2}$ to $5 \mathrm{mmHg}$ below the baseline value while breathing $\mathrm{O}_{2}$ to increase $\mathrm{ETO}_{2}$ to $400 \mathrm{mmHg}$. A marked decrement in $\mathrm{CBF}$ is evident globally. In image $\mathrm{C}$, the difference map (image 2-image 1) is shown indicating the regional decrease in $\mathrm{CBF}$ throughout the entire brain. See reference 5 for details of the methodology. Cerebral blood flow is shown as $\mathrm{mL} \cdot 100 \mathrm{~g}^{-1} \cdot \mathrm{min}^{-1}$. The number to the left and below each image is the distance above or below the anterior commissure-posterior commissure (AC-PC) line for that axial slice. $\mathrm{CBF}=$ cerebral blood flow. examining the association of any anesthetic agent with POD. Future clinical research should conceivably better leverage these data streams of ET vapour, $\mathrm{O}_{2}$ and $\mathrm{CO}_{2}$, blood pressure, pharmacologic blood concentration modelling of intravenous anesthetic agents, and serial blood gases and hemoglobin. A comprehensive understanding of contributing factors may allow the development of more effective preventative strategies.

We believe that clinical practice may benefit from an appraisal of how carbon dioxide elimination and oxygen delivery affect $\mathrm{CBF}$ and their potential influence on postoperative cerebral health including POND. In this light, we have registered a pilot study at www. clinicaltrials.gov (NCT04406350) to investigate the feasibility and safety of such an approach.

Disclosures None.

Funding statement Anesthesia Oversight Committee, Department of Anesthesiology, Perioperative and Pain Medicine, University of Manitoba.

Editorial responsibility This submission was handled by Dr. Gregory L. Bryson, Deputy Editor-in-Chief, Canadian Journal of Anesthesia.

\section{References}

1. Mutch WA, El-Gabalawy $R$. Anesthesia and postoperative delirium: the agent is a strawman - the problem is $\mathrm{CO}_{2}$. Can $\mathrm{J}$ Anesth 2017; 64: 678-80.

2. Evered L, Silbert B, Knopman DS, et al. Recommendations for the nomenclature of cognitive change associated with anaesthesia and surgery-2018. Can J Anesth 2018; 65: 1248-57.

3. Mutch WA, El-Gabalawy R, Girling L, Kilborn K, Jacobsohn E. End-tidal hypocapnia under anesthesia predicts postoperative delirium. Front Neurol 2018. https://doi.org/10.3389/fneur.2018. 00678.

4. Joosten A, Rinehart J, Bardaji A, et al. Anesthetic management using multiple closed-loop systems and delayed neurocognitive recovery: a randomized controlled trial. Anesthesiology 2020; 132: 253-66.

5. Mutch WA, El-Gabalawy R, Ryner L, et al. Brain BOLD MRI $\mathrm{O}_{2}$ and $\mathrm{CO}_{2}$ stress testing: implications for perioperative neurocognitive disorder following surgery. Crit Care 2020; 76. DOI: https://doi.org/10.1186/s13054-020-2800-3

6. Akkermans A, van Waes JA, Thompson A, et al. An observational study of end-tidal carbon dioxide trends in general anesthesia. Can J Anesth 2019; 66: 149-60.

Publisher's Note Springer Nature remains neutral with regard to jurisdictional claims in published maps and institutional affiliations. 